\title{
Effectiveness and safety of high dose clopidogrel plus aspirin in ischemic stroke patients with the single CYP2C19 loss-of- function allele: a randomized trial
}

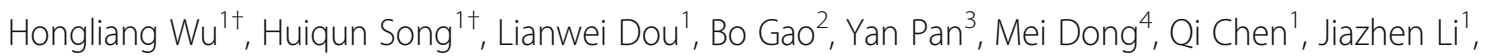
Lixiang Song ${ }^{1}$, Chuanyu Liu', Bing Li ${ }^{1}$ and Wenzheng Chu*

\begin{abstract}
Background: Dual antiplatelet aggregation therapy leads to better outcomes in patients with carotid artery stenosis, intracranial artery stenosis, minor strokes, or transient ischaemic attacks. However, carriers of the CYP2C19 loss-of-function allele may not experience the desired effects. We attempted to increase the clopidogrel dose to determine whether it would improve the outcomes of stroke patients who carry a single loss-of-function allele.

Methods: We recruited 131 patients with minor ischaemic stroke, within less than 7 days of stroke onset and a CYP2C19 loss-of-function allele, who had moderate-to-severe cerebral artery stenosis. Patients were divided into the high dose group (clopidogrel $150 \mathrm{mg}$ per day + aspirin $100 \mathrm{mg}$ per day over 21 days.) and a normal dose group (clopidogrel $75 \mathrm{mg}$ per day + aspirin $100 \mathrm{mg}$ per day over 21 days). The reported outcomes included any vascular or major bleeding events as the primary and safety endpoints, respectively.

Results: One and six vascular events occurred in the high dose and normal dose groups during the 3-months follow-up period, respectively. However, no significant difference was found between the two groups when adjusted for history of diabetes (hazard ratio, 5482; $95 \%$ confidence interval, 0.660 to $45.543 ; P=0.115$ ). No major bleeding events occurred.
\end{abstract}

Conclusions: In patients with ischaemic stroke who had a single CYP2C19 loss-of-function allele and moderate to severe cerebral stenosis, fewer vascular events occurred within 3 months with high dose of clopidogrel and aspirin than with normal dose of clopidogrel and aspirin. However, the difference between the two groups was not significant.

Trial registration: Clinical study of clopidogrel in the treatment of patients with symptomatic moderate to severe cerebral artery stenosis with intermediate metabolites of CYP2C19, URL: http://www.chictr.org.cn/. Unique identifier: ChiCTR1800017411, 07/28/2018;

Keywords: Ischaemic stroke, Intracranial stenosis, Carotid stenosis, CYP2C19, Clopidogrel, Dual antiplatelet aggregation therapy

\footnotetext{
* Correspondence: chuwz2020@outlook.com

${ }^{\dagger}$ Hongliang Wu and Huiqun Song contributed equally to this work.

'Department of Neurology, The Affiliated Yantai Yuhuangding Hospital of

Qingdao University, Yantai, Shandong 264000, P.R. China

Full list of author information is available at the end of the article
}

(c) The Author(s). 2020 Open Access This article is licensed under a Creative Commons Attribution 4.0 International License, which permits use, sharing, adaptation, distribution and reproduction in any medium or format, as long as you give appropriate credit to the original author(s) and the source, provide a link to the Creative Commons licence, and indicate if changes were made. The images or other third party material in this article are included in the article's Creative Commons licence, unless indicated otherwise in a credit line to the material. If material is not included in the article's Creative Commons licence and your intended use is not permitted by statutory regulation or exceeds the permitted use, you will need to obtain permission directly from the copyright holder. To view a copy of this licence, visit http://creativecommons.org/licenses/by/4.0/ The Creative Commons Public Domain Dedication waiver (http://creativecommons.org/publicdomain/zero/1.0/) applies to the data made available in this article, unless otherwise stated in a credit line to the data. 


\section{Background}

Stenosis of the cerebral blood supply arteries, including intracranial atherosclerotic stenosis or extracranial carotid stenosis, is a major contributing factor to ischaemic stroke and stroke recurrence. Several studies have shown that dual antiplatelet aggregation therapy is an effective treatment. Patients in the SAMMPRIS trial [1] achieved a better prognosis than those in the WASID trial, with aspirin and clopidogrel for 90 consecutive days while actively controlling lipid, blood pressure, and glucose levels. In another study [2], patients with intracranial artery stenosis who received double antiplatelet aggregation therapy with aspirin and clopidogrel within less than 7 days of onset were significantly less likely to have a microembolic signal detected on transcranial Doppler (TCD) than those who were administered only aspirin. In a study of symptomatic carotid stenosis [3], coadministration of clopidogrel and aspirin significantly reduced the occurrence of symptomatic embolism compared to the administration of only aspirin. In a trial of transient cerebral ischaemia and minor stroke [4], co-administration of clopidogrel and aspirin reduced the risk of recurrent stroke within 90 days compared to the administration of only aspirin, without increasing the risk of bleeding. However, in this trial, dual antiplatelet aggregation was superior to aspirin alone only in patients without CYP2C19 loss-offunction allele (LoFA), which was present in $58.8 \%$ of patients [5].

Clopidogrel is biotransformed by cytochrome P-450 enzymes into an active metabolite. Patients carrying CYP2C19 LoFAs have lower levels of clopidogrel active metabolites [6]. In East Asian populations, the most common LoFAs are CYP2C19:2 (frequency of 30-50\%) and CYP2C19*3 (frequency of 5-10\%) [7]. Carriers of these two LoFAs have a decreased platelet response to clopidogrel and an elevated cardiovascular disease risk [8-11]. Some studies have attempted to increase clopidogrel doses to treat ischaemic disease. In a trial of patients with acute coronary syndrome who were being prepared for percutaneous coronary intervention (PCI), randomization to double-dose clopidogrel $(150 \mathrm{mg}$ per day) had a lower risk of cardiovascular events and instent thrombosis than the standard dose $(75 \mathrm{mg}$ per day). This treatment increased the risk of bleeding, but not of fatal bleeding or intracranial hemorrhage [12]. This study did not select patients based on metabolic genes, which may have contributed to increased bleeding. In another trial [13], triple-dose clopidogrel (225 mg per day) enabled $C Y P 2 C 19 * 2$ heterozygotes to achieve platelet responsiveness at the level of normal doses (75 mg per day) for non-carriers. In contrast, for CYP2C19*2 homozygotes, the corresponding level of platelet inhibition was not achieved even at $300 \mathrm{mg} /$ day. We speculate that for CYP2C19 " $1 / * 1$ and *1/*17 carriers, higher doses of clopidogrel may lead to increased bleeding, whereas for two LoFA carriers, higher doses do not improve antiplatelet effects. In addition, Kobayashi et al. found that among healthy Japanese subjects, intermediate metabolic subjects who were administered a 7-day double dose of clopidogrel achieved an antiplatelet effect close to that of fast metabolic subjects and was well tolerated [14].

Based on these results, we selected patients with moderately severe cerebrovascular stenosis within less than 7 days of cerebral infarction onset, who had only one CYP2C19 LoFA to understand the effectiveness and safety of double-dose clopidogrel administration.

Our study adheres to CONSORT guidelines.

\section{Methods}

This is a prospective, single-centre, parallel-group, randomized, superiority trial. The study protocol was approved by the Ethics Committee of Yantai Yuhuangding Hospital (approval no. 193: [2017]). All patients provided informed consent prior to enrolment.

\section{Study population Inclusion criteria}

Patients with acute ischaemic stroke who are continuously hospitalised; aged $\geq 40$ years and $\leq 75$ years; with moderate to severe cerebral artery stenosis (stenosis > $50 \%$ ) within less than 7 days of ischaemic stroke onset and access to the study drug within $24 \mathrm{~h}$ of admission; patients with a single CYP2C19 LoFA ("1/*2, "1/*3).

After trial commencement, we added National Institutes of Health Stroke Scale (NIHSS) score $\leq 5$ as an inclusion criterion to decrease the risk of cerebral haemorrhage.

\section{Exclusion criteria}

The present attack was confirmed as a non-cerebrovascular attack by cranial magnetic resonance imaging or cranial computed tomography; significant signs of anticoagulation were present (suspected cardiogenic embolism, such as rheumatic heart valve disease, known artificial heart valves, atrial fibrillation, and suspected endocarditis); bleeding from the gastrointestinal tract within 1 year, or positive faecal occult blood on admission to hospital; previous history of intracranial haemorrhage (cerebral haemorrhage or subarachnoid haemorrhage); severe heart failure, asthma, liver, and kidney insufficiency; previous history of coagulation abnormalities or systemic bleeding disorders, previous history of hemocytopenia, leukopoenia $\left(<2 \times 10^{9} / \mathrm{L}\right)$, or thrombocytopenia $\left(<100 \times 10^{9} / \mathrm{L}\right)$; patients who were given aspirin combined with clopidogrel therapy at randomisation; patients who were pregnant or participating in other clinical trials; and any patients or their legal representative who refused to participate in the investigation. 


\section{Determination of arterial stenosis}

Extracranial carotid artery (including internal and common carotid arteries) stenosis was determined by carotid Doppler sonography studies, which were performed by the same diagnostic sonographer in our hospital's ultrasound department. The degree of stenosis was determined by a combined definition of peak systolic velocities, enddiastolic velocities, and B-mode [15].

Intracranial artery stenosis was determined by head magnetic resonance angiography (MRA). All MRAs were analyzed by the same diagnostic imaging physician. The degree of stenosis is determined by the narrowest diameter of the stenosis versus the normal vessel diameter of the same artery. The vessels included in this study were: the internal carotid artery intracranial segment, the middle cerebral artery M1 segment, the vertebral artery intracranial segment, and the basilar artery.

Those with presence of moderate to severe stenosis $(\geq 50 \%)$ in the above vessels and infarct lesion located in the blood supply area of the stenotic vessel were eligible for inclusion in this study.

\section{Clinical data collection}

We collected baseline data including: demographic data, past disease history, blood pressure, blood cell counts, fasting blood glucose, lipid series, bilirubin, transaminases, creatinine, urea, homocysteine, glycosylated haemoglobin, and uric acid. We routinely evaluated patients using the modified Rankin Scale (MRS) and the NIHSS score on admission and discharge.

We collected the above data from the hospital's medical records system at patients' discharge time.

\section{Treatment}

Eligible patients were randomly assigned to treatment groups in a 1:1 ratio. The high dose group was administered $150 \mathrm{mg}$ of clopidogrel and $100 \mathrm{mg}$ of aspirin per day after initially receiving $300 \mathrm{mg}$ of clopidogrel on day 1 , while the normal dose group was given $75 \mathrm{mg}$ of clopidogrel and $100 \mathrm{mg}$ of aspirin per day after initially receiving $300 \mathrm{mg}$ of clopidogrel on day 1 . All patients were maintained on dual antiplatelet aggregation therapy for 21 days according to the two different regimens; then clopidogrel was discontinued, and aspirin $100 \mathrm{mg}$ daily was continued throughout the observation period (90 days from stroke onset).

All patients were administered pantoprazole $40 \mathrm{mg}$ per day during dual antiplatelet therapy and atorvastatin 20 mg per day throughout the observation period (90 days from stroke onset).

\section{Sample size and randomization}

No previous studies have provided information on the incidence of vascular events within 90 days of mild stroke associated with cerebral stenosis. We estimated the incidence of vascular events in the control group based on data from previous trials $[1,4]$ to be greater than $20 \%$, while the incidence in the high dose group was less than $5 \%$. Taking the alpha value of 0.05 , We decided to recruit at least 130 patients in total. We applied the simple randomization method to divide the recruited patients into the high dose group and the normal dose group in a 1:1 ratio.

\section{Endpoint}

Information regarding vascular events during the followup was obtained for all patients through standard telephone interviews. The primary endpoint for the trial was the time from randomisation to the first vascular event within 3 months of the follow-up. A vascular event was defined as any stroke (including ischaemic or haemorrhagic), myocardial infarction, or death. The secondary endpoint was the neurological outcome at discharge, as assessed by the NIHSS score.

\section{Safety evaluation}

The safety endpoint was defined as the time from randomization to a major bleeding event or any bleeding event that resulted in the trial treatment being discontinued, such as intracranial haemorrhage, gastrointestinal haemorrhage, haemoptysis, and pericardial occlusion.

\section{Statistical analysis}

Baseline characteristics were compared between the high dose and normal dose groups. Categorical variables were displayed as percentages, and chi-square tests were used. Normally distributed continuous variables were presented as mean \pm standard deviation and compared using analysis of variance (ANOVA). Continuous variables with abnormal distributions were presented as medians (interquartile ranges) and compared using the nonparametric Wilcoxon test between groups. The NIHSS scores were compared between the two groups by the nonparametric Wilcoxon test. Survival plots were generated by applying the KaplanMeier product limit method, and differences between treatment groups were tested for significance by the logrank test. Cox regression models were used to assess the associations between treatment groups and vascular events during the 90-day follow-up period and adjusted based on the history of diabetes mellitus. All statistical analyses were performed using STATA (version 14.0; StataCorp LLC, College Station, TX, USA).

\section{Results}

Overall, 162 patients were enrolled in this study from August 2018 to February 2019 and were followed up for a 90-day period, 154 patients underwent randomization, with 76 being placed in the high dose group and 78 in 
the normal dose group (Fig. 1). A total of 18 patients did not receive the trial intervention because of NIHSS $>5$ or bleeding from any part of the body; 11 in the high dose group, and 7 in the normal dose group. Five patients were lost during follow-up because of invalid contact information, 3 in the high dose group, and 2 in the normal dose group. Ultimately, 131 patients were included in the analysis. Sixty-two patients were finally included in the high dose group and 69 in the normal dose group. Table 1 shows the baseline characteristics between the high dose and normal dose groups. The proportion of patients with a history of diabetes was significantly higher in the normal dose group than in the high dose group, but no difference in fasting glucose or glycosylated haemoglobin was observed between the two groups. Other baseline data such as sex, age, blood pressure, lipids, haemoglobin, and platelets did not differ between the two groups.

The NIHSS scores at admission and discharge were not significantly different between the two groups. (Fig. 2). Of the 131 patients who completed the 90-day follow-up, seven had vascular events. Of these, one was in the high dose group (1.64\%), and six were in the normal dose group (8.82\%). The Kaplan-Meier survival estimates are shown in Fig. 3. In the log-rank test, the two groups were not significantly different from each other $(p=0.0763)$. The risk of vascular events within 90 days was not significantly different between the two groups in the Cox regression analysis (Table 2). Considering that the two groups differed in the prevalence of patients with history of diabetes, we added a history of diabetes to the adjustment and the risk of vascular events remained insignificantly different between the two groups.

One patient in the normal dose group died of recurrent cerebral infarction within 90 days, and one patient in the high dose group was found to have subcutaneous haemorrhage. Ischaemic cerebrovascular disease recurred in one patient in the high dose group compared to three patients in the normal dose group, and angina was seen in two patients in the normal dose group. Patients in both groups did not experience intracranial haemorrhage, gastrointestinal haemorrhage, haemoptysis, pericardial occlusion, or other bleeding events leading to anaemia.

\section{Discussion}

In this study, we enrolled patients with a minor acute ischaemic stroke within less than 7 days of onset (NIHSS $\leq 5$ ) and moderate to severe stenosis of the cerebral blood supply arteries. Based on previous findings, these patients may benefit from dual antiplatelet aggregation therapy [1-4]. However, drug resistance may affect treatment outcomes. Activity of drug metabolism gene, CYP2C19, is an important factor in treatment outcomes. The selected intermediate metabolic gene carriers $\left({ }^{*} 1 /{ }^{*} 2\right.$, $* 1 / 33$ in this study may not respond adequately to normal doses of clopidogrel [5], and increased doses may result in better outcomes [13, 14]. In this study, the high dose group did have fewer patients with vascular events within 90 days than the normal dose group, but this difference was not statistically significant.

Studies on the antiplatelet aggregation function and clinical effects of high-dose clopidogrel in CYP2C19 genotype patients receiving $\mathrm{PCI}$ have presented conflicting

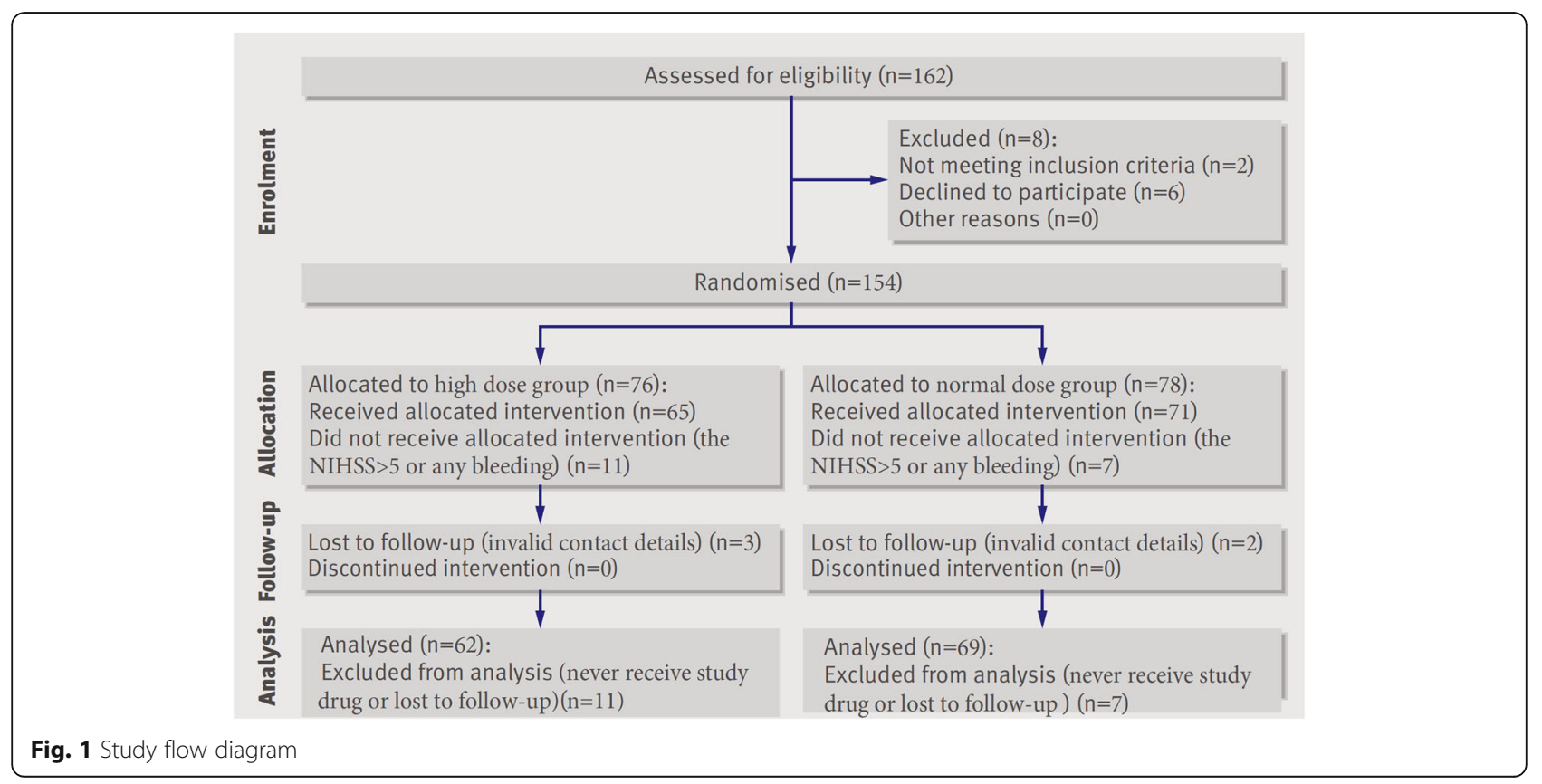


Table 1 Comparison of the baseline data between the two groups of patients

\begin{tabular}{|c|c|c|c|}
\hline & High dose group & Normal dose group & $p$-value \\
\hline Gender, n (male/\%) & $49(79.03)$ & $50(72.46)$ & 0.381 \\
\hline H. Stroke, n (\%) & $12(19.35)$ & $11(15.94)$ & 0.608 \\
\hline CHD, n (\%) & $7(11.29)$ & $8(11.59)$ & 0.956 \\
\hline $\mathrm{DM}, \mathrm{n}(\%)$ & $18(29.03)$ & $32(46.38)$ & 0.040 \\
\hline Hypertension, n (\%) & $44(70.97)$ & $49(71.01)$ & 0.995 \\
\hline Smoker, n (\%) & $31(50.00)$ & $33(47.83)$ & 0.804 \\
\hline Drinker, n (\%) & $26(41.94)$ & $28(40.58)$ & 0.875 \\
\hline Age, median (IQR) & $60.0 \pm 10.4$ & $63.2 \pm 9.3$ & 0.406 \\
\hline $\mathrm{SBP}, \mathrm{mmHg} \pm \mathrm{SD}$ & $148(133-162)$ & $151(139-167)$ & 0.390 \\
\hline $\mathrm{DBP}, \mathrm{mmHg} \pm \mathrm{SD}$ & $88(78-95)$ & 86 (79-96) & 0.854 \\
\hline FBG, mmol/L, median (IQR) & $5.18(4.49-6.95)$ & $5.54(4.67-7.59)$ & 0.178 \\
\hline $\mathrm{GH}, \%$, median (IQR) & $5.9(5.6-6.9)$ & $6.1(5.6-8.0)$ & 0.409 \\
\hline $\mathrm{HCY}$, umol/L, median (IQR) & $12.1(10.5-13.9)$ & $11.9(10.5-14.5)$ & 0.921 \\
\hline $\mathrm{PLT}, \times 10^{9} / \mathrm{L}$, median (IQR) & $229(199-254)$ & $217(180-235)$ & 0.402 \\
\hline HGB, g/L, median (IQR) & $148(140-158)$ & $141(132-153)$ & 0.423 \\
\hline $\mathrm{CHL}, \mathrm{mmol} / \mathrm{L}$, median (IQR) & $4.27(3.69-5.43)$ & $4.02(3.33-4.82)$ & 0.332 \\
\hline $\mathrm{HDL}, \mathrm{mmol} / \mathrm{L}$, median (IQR) & $1.09(0.94-1.26)$ & $1.11(0.92-1.27)$ & 0.747 \\
\hline LDL, mmol/L, median (IQR) & $2.47(1.89-3.22)$ & $2.21(1.75-2.86)$ & 0.970 \\
\hline TG, mmol/L, median (IQR) & $1.28(0.98-1.86)$ & $1.30(0.96-1.55)$ & 0.627 \\
\hline AST U/L, median (IQR) & $20.0(16.0-26.0)$ & $19.0(16.0-25.0)$ & 0.943 \\
\hline CRE, umol/L, median (IQR) & $69.5(57.0-77.0)$ & $67.0(55.0-84.0)$ & 0.655 \\
\hline URE, mmol/L, median (IQR) & $5.15(4.34-6.07)$ & $4.70(4.04-5.74)$ & 0.211 \\
\hline UA, umol/L, median (IQR) & $318.1 \pm 89.7$ & $311.1 \pm 79.7$ & 0.341 \\
\hline
\end{tabular}

H. stroke History of stroke, CHD Coronary atherosclerotic heart disease, DM History of diabetes mellitus, SBP Systolic blood pressure, DBP Diastolic blood pressure, FBG Fasting blood glucose, GH Glycosylated haemoglobin, $H C Y$ Homocysteine, PLT Platelets, HGB Haemoglobin, $C H L$ Cholesterol, $H D L$ High-density lipoprotein, $L D L$ Low-density lipoprotein, TG Triglyceride, AST Aspartate aminotransferase, CRE Creatinine, URE Urea, UA Uric acid

results. It was found that a higher dose of clopidogrel results in better platelet inhibitory effects in patients with one LoFA, but patients with two or more LoFAs do not achieve the same degree of platelet inhibition [13, 16, 17]. In addition, other studies have found that increased doses of clopidogrel do not lead to better results $[18,19]$. In the ARCTIC-GENE study [18], the investigators did not distinguish between homogeneous and heterogeneous CYP2C19*2 as a possible reason why increasing the clopidogrel dose still did not improve outcomes. In the GRAV

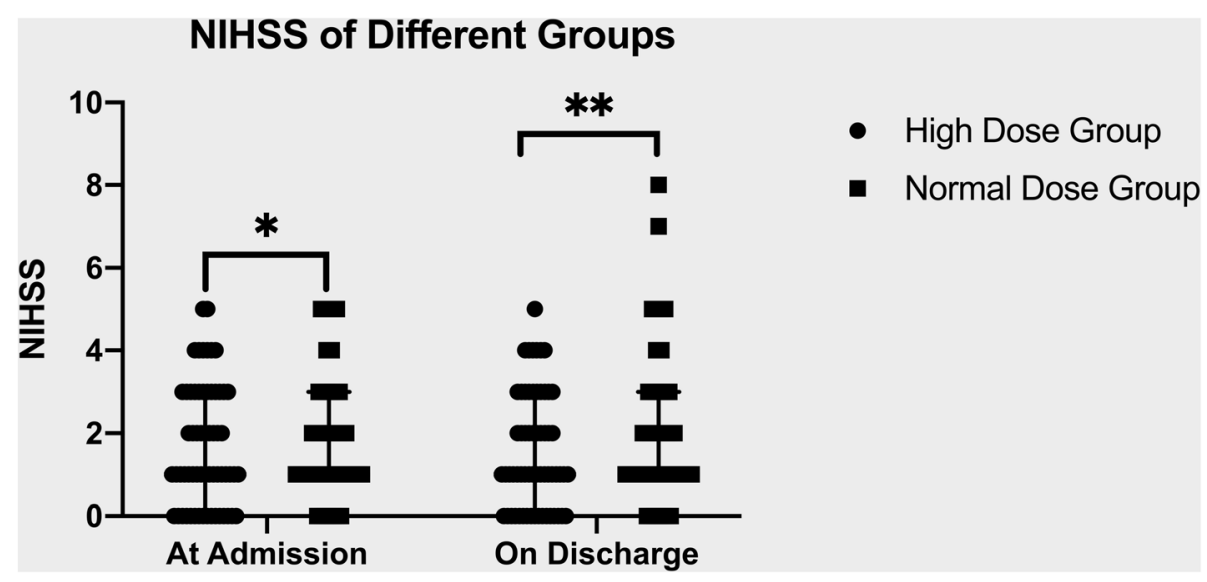

Fig. 2 Mann-Whitney test, ${ }^{*} p=0.5286 * * 0.2192$. NIHSS indicates National Institutes of Health stroke scale 


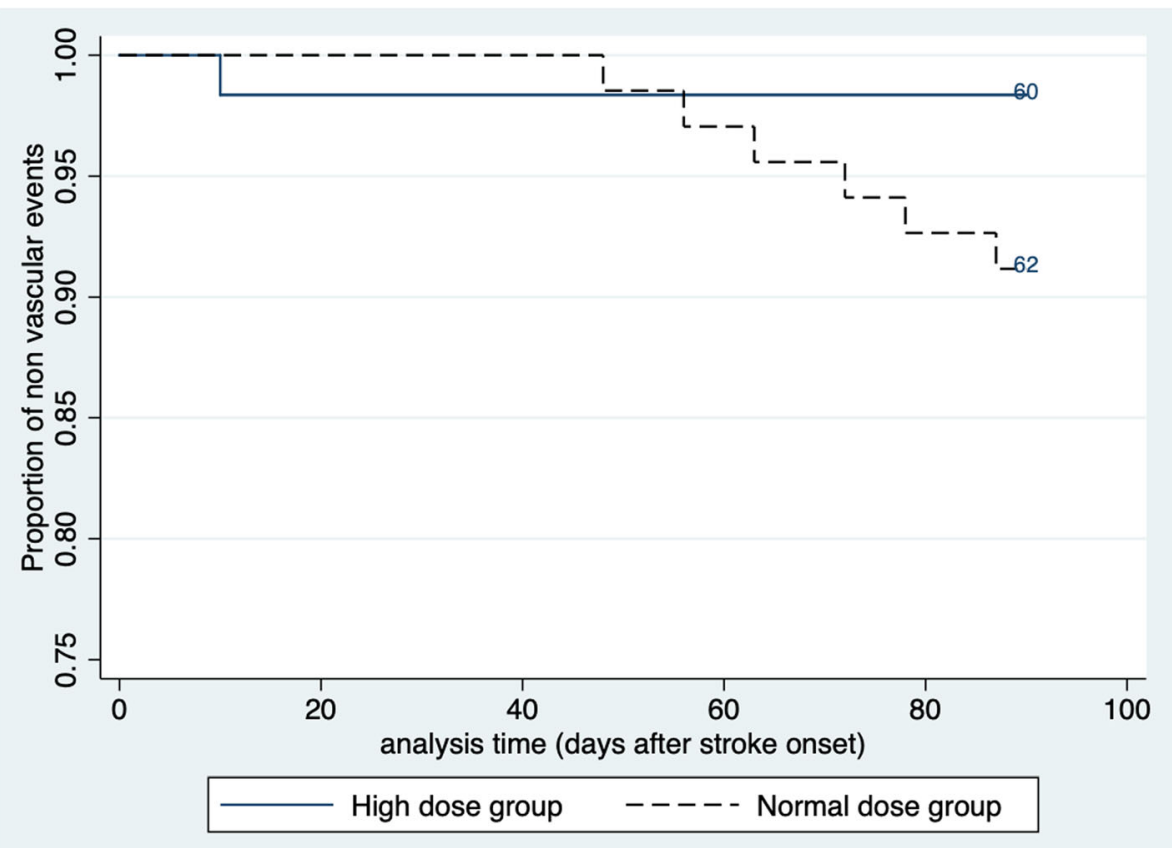

Fig. 3 Kaplan-Meier survival estimates

ITAS randomised trial [19], increasing the clopidogrel dose in patients with high treatment responsiveness as tested by VerifyNow P2Y12, did not clinically improve results compared to the standard-dose treatment group, but there were no more cases of bleeding. Metabolic genes were not taken into account in this study, and the fact that patients carrying LoFAs may not achieve improved clinical outcomes even with increased doses may be a possible reason for the negative trial results. An analysis of the above studies found that patients with intermediate metabolic patterns (only one LoFA) may benefit from clopidogrel dosing.

Studies with adjusted clopidogrel dosage or administration of an alternative drug by measuring platelet aggregation function or rate of inhibition (vasodilator stimulated phosphoprotein phosphorylation assay [VASP] or verifyNOW) have not demonstrated efficacy of therapeutic adjustment [20-22]. In addition, the existing methods for measuring platelet aggregation function include VASP, multiplate impedance aggregation (MP) and light transmission aggregation (LTA), etc. However, the

Table 2 Effect of high dose clopidogrel versus normal dose clopidogrel on vascular events

\begin{tabular}{|c|c|c|c|}
\hline & Hazard Ratio & 95\% Conf. Interval & $p$-value \\
\hline Different groups & 5.482 & $0.660-45.543$ & 0.115 \\
\hline \multicolumn{4}{|c|}{ Adjusted by diabetes mellitus history } \\
\hline Different groups & 5.001 & $0.595-42.177$ & 0.139 \\
\hline Diabetes mellitus & 1.746 & $0.387-7.887$ & 0.469 \\
\hline
\end{tabular}

lack of consistency between different platelet function tests makes it difficult to choose the appropriate test method [23]. Therefore, platelet function testing was not performed for each patient in this study.

Few studies have addressed the dose adjustment of clopidogrel for ischaemic stroke. Some investigators have found that administering $300 \mathrm{mg}$ clopidogrel [24, 25] or $375 \mathrm{mg}$ clopidogrel [26] for the first dose to patients with ischaemic stroke does not add to the bleeding risk. One study found [25] that administration of a loading dose improved neurological function in cerebral infarction patients 3 months after onset. A double maintenance dose of clopidogrel has not been studied in patients with ischaemic stroke. We excluded patients with normal metabolism of CYP2C19 (*1/*1) to avoid an increased bleeding risk. In addition, we excluded patients with two LoFAs based on previous studies $[13,16,17]$ to reduce the fact that increasing the dose did not increase the efficacy. There were no serious bleeding events in this study, suggesting that it may be safe to increase the dose of clopidogrel in this group of patients. In addition, the high dose group had fewer patients with vascular events within 3 months than the normal dose group, but the results did not reach statistical significance.

Other researchers have investigated alternatives to clopidogrel. A Japanese study found that prasugrel and clopidogrel were equally effective at preventing vascular events in non-cardioembolic stroke patients [27, 28]. A Chinese study found that ticagrelor plus aspirin treated patients with transient ischaemic attack (TIA) or minor 
stroke had lower platelet activity than those treated with clopidogrel, especially CYP2C19 LoFA carriers [29]. Clopidogrel is more economical and accessible than the two drugs mentioned above, and if better efficacy is obtained in screened patients by increasing the dose, better societal benefits can be obtained.

In this study, the two groups had different proportions of patients with a history of diabetes, which may have influenced the final results. Diabetes may affect the response to clopidogrel in patients with cerebrovascular disease. There are many reasons for poor responsiveness to clopidogrel in patients with diabetes, including metabolic, genetic, and other clinical factors [30-32]. However, it has also been shown that clopidogrel has a consistent effect on platelet inhibition in both diabetic and non-diabetic patients [33]. In a study of metabolic genes, diabetic $C Y P 2 C 19 * 2$ carriers were found to have a poorer response to clopidogrel than diabetic noncarriers, while non-diabetics showed no such difference [34]. In another study, both CYP2C19*2 carrier and noncarrier diabetic patients had poorer responses to clopidogrel than non-diabetic patients, but there was no difference between the two, and non-diabetic CYP2C19*2 carriers had higher platelet reactivity than non-carriers after clopidogrel application [35]. In the present study, when adjusted for diabetes, the results did not change.

There are several limitations in this study. First of all, this is an open-label study in only one academic stroke centre, and the results should be carefully interpreted. Second, the patients and investigators were not blinded, which may have introduced bias in the outcome assessments. Third, the sample size was small, and the proportion of patients with diabetes was different between the two groups, indicating that the basic characteristics of the two groups of patients were not completely consistent, which may have affected the results. We adjusted for diabetes in the final comparison of the results. Despite these limitations, the absence of serious bleeding events in our study suggests that larger and more rigorous clinical trials may be attempted to understand the effectiveness and safety of high dose clopidogrel therapy.

\section{Conclusion}

In patients with ischaemic stroke who had only one CYP2C19 LoFA and moderate to severe cerebral stenosis, fewer vascular events occurred within 3 months with high dose clopidogrel combined with aspirin than with normal dose clopidogrel combined with aspirin. However, no significant difference was observed between the two groups. In addition, neither the high nor the normal dose group experienced bleeding events.

\section{Abbreviations}

SAMMPRIS: Stenting and Aggressive Medical management for prevention of Recurrent Stroke in Intracranial Stenosis trial; WASID: Warfarin Aspirin
Symptomatic Intracranial Disease trial; TCD: Transcranial Doppler; LoFA: Lossof-function allele; PCl: Percutaneous coronary intervention; NIHSS: National Institutes of Health Stroke Scale; MRS: Modified Rankin Scale; TIA: Transient ischaemic attack; CYP: Cytochrome P-450

\section{Acknowledgments}

We would like to thank Editage (www.editage.com) for English language editing.

\section{Authors' contributions}

HLW designed and conceptualised the study; acquisition of the data; analysed the data; drafted the manuscript for intellectual content. HQS acquisition of the data in hospital; generated the random allocation sequence; revised the manuscript for intellectual content. LWD acquisition of data in hospital; BG interpreted the magnetic resonance imaging. YP completed all carotid ultrasounds. MD analysed the CYP2C19 gene assay. QC acquisition of the data in hospital. JZL and LXS collected the 30-day and 90day follow-up data. BL and CYL designed the study and determined whether patients met the recruitment criteria, including initial data collection. WZC designed and conceptualised the study; acquisition of data; analysed the data; revised the manuscript for intellectual content. All authors have read and approved the manuscript.

\section{Funding}

This study was funded by Shandong Province Medical and Health Science and Technology Development Plan Project 2017WS168 and Yantai Yuhuangding Hospital Youth Research Initiation Fund. However, the funders had no role in the study design, data collection and analysis, decision to publish, or preparation of the manuscript.

\section{Availability of data and materials}

The datasets used and/or analysed during the current study are available from the corresponding author upon reasonable request.

\section{Ethics approval}

The study protocol was approved by the Ethics Committee of Yantai Yuhuangding Hospital (approval no. 193: [2017 -]). All patients provided written informed consent to participate before enrolment.

\section{Consent for publication}

Not Applicable.

\section{Competing interests}

No potential conflicts of interest relevant to this article were reported. There are no competing interests to declare.

\section{Author details}

'Department of Neurology, The Affiliated Yantai Yuhuangding Hospital of Qingdao University, Yantai, Shandong 264000, P.R. China. ${ }^{2}$ Department of Radiology, The Affiliated Yantai Yuhuangding Hospital of Qingdao University, Yantai, Shandong 264000, P.R. China. ${ }^{3}$ Department of Ultrasound, The Affiliated Yantai Yuhuangding Hospital of Qingdao University, Yantai, Shandong 264000, P.R. China. ${ }^{4}$ Department of Cardiology, The Affiliated Yantai Yuhuangding Hospital of Qingdao University, Yantai, Shandong 264000, P.R. China.

Received: 11 July 2020 Accepted: 23 October 2020

Published online: 29 October 2020

\section{References}

1. Chimowitz MI, Lynn MJ, Derdeyn CP, Turan TN, Fiorella D, Lane BF, Janis LS, Lutsep HL, Barnwell SL, Waters MF, et al. Stenting versus aggressive medical therapy for intracranial arterial stenosis. N Engl J Med. 2011;365(11):993-1003.

2. Wang $X$, Lin WH, Zhao YD, Chen XY, Leung TW, Chen C, Fu J, Markus H, Hao Q, Wong KS, et al. The effectiveness of dual antiplatelet treatment in acute ischemic stroke patients with intracranial arterial stenosis: a subgroup analysis of CLAIR study. Int J Stroke. 2013;8(8):663-8.

3. Markus HS, Droste DW, Kaps M, Larrue V, Lees KR, Siebler M, Ringelstein EB. Dual antiplatelet therapy with clopidogrel and aspirin in symptomatic carotid stenosis evaluated using doppler embolic signal detection: the 
Clopidogrel and aspirin for reduction of emboli in symptomatic carotid stenosis (CARESS) trial. Circulation. 2005;111(17):2233-40.

4. Wang Y, Wang Y, Zhao X, Liu L, Wang D, Wang C, Wang C, Li H, Meng X, Cui $L$, et al. Clopidogrel with aspirin in acute minor stroke or transient ischemic attack. N Engl J Med. 2013;369(1):11-9.

5. Wang Y, Zhao X, Lin J, Li H, Johnston SC, Lin Y, Pan Y, Liu L, Wang D, Wang C, et al. Association between CYP2C19 loss-of-function allele status and efficacy of Clopidogrel for risk reduction among patients with minor stroke or transient ischemic attack. JAMA. 2016;316(1):70-8.

6. Hulot JS, Bura A, Villard E, Azizi M, Remones V, Goyenvalle C, Aiach M, Lechat P, Gaussem P. Cytochrome P450 2 C19 loss-of-function polymorphism is a major determinant of clopidogrel responsiveness in healthy subjects. Blood. 2006;108(7):2244-7.

7. Xie HG, Zou JJ, Hu ZY, Zhang JJ, Ye F, Chen SL. Individual variability in the disposition of and response to clopidogrel: pharmacogenomics and beyond. Pharmacol Ther. 2011;129(3):267-89.

8. Zou JJ, Xie HG, Chen SL, Tan J, Lin L, Zhao YY, Xu HM, Lin S, Zhang J, Wang GJ. Influence of CYP2C19 loss-of-function variants on the antiplatelet effects and cardiovascular events in clopidogrel-treated Chinese patients undergoing percutaneous coronary intervention. Eur J Clin Pharmacol. 2013; 69(4):771-7.

9. Yang J, Zhao HD, Tan J, Ding YL, Gu ZQ, Zou JJ. CYP2C19 polymorphism and antiplatelet effects of clopidogrel in Chinese stroke patients. Pharmazie. 2013;68(3):183-6.

10. Simon T, Verstuyft C, Mary-Krause M, Quteineh L, Drouet E, Meneveau N, Steg PG, Ferrieres J, Danchin N, Becquemont L, et al. Genetic determinants of response to clopidogrel and cardiovascular events. N Engl J Med. 2009; 360(4):363-75.

11. Shuldiner AR, O'Connell JR, Bliden KP, Gandhi A, Ryan K, Horenstein RB, Damcott CM, Pakyz R, Tantry US, Gibson Q, et al. Association of cytochrome P450 2C19 genotype with the antiplatelet effect and clinical efficacy of clopidogrel therapy. JAMA. 2009;302(8):849-57.

12. Mehta SR, Tanguay JF, Eikelboom JW, Jolly SS, Joyner CD, Granger CB, Faxon DP, Rupprecht HJ, Budaj A, Avezum A, et al. Double-dose versus standard-dose clopidogrel and high-dose versus low-dose aspirin in individuals undergoing percutaneous coronary intervention for acute coronary syndromes (CURRENT-OASIS 7): a randomised factorial trial. Lancet. 2010;376(9748):1233-43.

13. Mega JL, Hochholzer W, Frelinger AL 3rd, Kluk MJ, Angiolillo DJ, Kereiakes DJ, Isserman S, Rogers WJ, Ruff CT, Contant C, et al. Dosing clopidogrel based on CYP2C19 genotype and the effect on platelet reactivity in patients with stable cardiovascular disease. JAMA. 2011;306(20):2221-8.

14. Kobayashi M, Kajiwara M, Hasegawa S. A randomized study of the safety, tolerability, pharmacodynamics, and pharmacokinetics of Clopidogrel in three different CYP2C19 genotype groups of healthy Japanese subjects. J Atheroscler Thromb. 2015;22(11):1186-96.

15. Taylor DC, Strandness DE Jr. Carotid artery duplex scanning. J Clin Ultrasound. 1987;15(9):635-44.

16. Jeong YH, Abadilla KA, Tantry US, Park Y, Koh JS, Kwak CH, Hwang JY, Gurbel PA. Influence of CYP2C19*2 and *3 loss-of-function alleles on the pharmacodynamic effects of standard- and high-dose clopidogrel in east Asians undergoing percutaneous coronary intervention: the results of the ACCEL-DOUBLE-2N3 study. J Thromb Haemost. 2013;11(6):1194-7.

17. Collet JP, Hulot JS, Anzaha G, Pena A, Chastre T, Caron C, Silvain J, Cayla G, Bellemain-Appaix A, Vignalou JB, et al. High doses of clopidogrel to overcome genetic resistance: the randomized crossover CLOVIS-2 (Clopidogrel and response variability investigation study 2). JACC Cardiovasc Interv. 2011:4(4):392-402

18. Collet JP, Hulot JS, Cuisset T, Range G, Cayla G, Van Belle E, Elhadad S, Rousseau H, Sabouret P, O'Connor SA, et al. Genetic and platelet function testing of antiplatelet therapy for percutaneous coronary intervention: the ARCTIC-GENE study. Eur J Clin Pharmacol. 2015;71(11):1315-24.

19. Price MJ, Berger PB, Teirstein PS, Tanguay JF, Angiolillo DJ, Spriggs D, Puri S, Robbins M, Garratt KN, Bertrand OF, et al. Standard- vs high-dose clopidogrel based on platelet function testing after percutaneous coronary intervention: the GRAVITAS randomized trial. JAMA. 2011:305(11):1097-105.

20. Collet JP, Cuisset T, Range G, Cayla G, Elhadad S, Pouillot C, Henry P, Motreff $P$, Carrie D, Boueri Z, et al. Bedside monitoring to adjust antiplatelet therapy for coronary stenting. N Engl J Med. 2012;367(22):2100-9.

21. Park Y, Jeong YH, Tantry US, Ahn JH, Kim KH, Koh JS, Park JR, Hwang SJ, Kwak CH, Hwang JY, et al. Effect of adjunctive dipyridamole to DAPT on platelet function profiles in stented patients with high platelet reactivity. The result of the ACCEL-DIP study. Thromb Haemost. 2014:112(6):1198-208.

22. Gonzalez A, Moniche F, Cayuela A, Gonzalez-Marcos JR, Mayol A, Montaner J. Antiplatelet effects of clopidogrel dose adjustment $(75 \mathrm{mg} / \mathrm{d}$ vs $150 \mathrm{mg} /$ d) after carotid stenting. J Vasc Surg. 2014;60(2):428-35.

23. Helten C, Naguib D, Dannenberg L, Pohl M, Ayhan A, Hohlfeld T, Levkau B, Kelm M, Zeus T, Polzin A. Platelet function testing: dead or alive. J Thromb Haemost. 2018;16(5):984-6.

24. Zhao Y, Yang W, Tan Z, Wang W, Xiao W, Zeng J, Xu A. Clopidogrel loading dose versus maintenance dose to treat patients with acute ischaemic stroke in China (CLASS-China): results from a prospective double-blind randomised clinical trial. Stroke Vasc Neurol. 2017;2(3):118-23.

25. Yang L, Diao SS, Ding YP, Huang SJ, Sun T, Lu Y, Fang Q, Cai XY, Kong Y, Xu $Z$. Efficacy and mechanism of loading dose clopidogrel in patients with transient ischemic attack and minor stroke. Zhonghua Yi Xue Za Zhi. 2019; 99(5):349-53.

26. Meyer DM, Albright KC, Allison TA, Grotta JC. LOAD: a pilot study of the safety of loading of aspirin and clopidogrel in acute ischemic stroke and transient ischemic attack. J Stroke Cerebrovasc Dis. 2008;17(1):26-9.

27. Ogawa A, Toyoda K, Kitagawa K, Kitazono T, Nagao T, Yamagami H, Uchiyama S, Tanahashi N, Matsumoto M, Minematsu K, et al. Comparison of prasugrel and clopidogrel in patients with non-cardioembolic ischaemic stroke: a phase 3, randomised, non-inferiority trial (PRASTRO-I). Lancet Neurol. 2019;18(3):238-47.

28. Kitagawa K, Toyoda K, Kitazono T, Nishikawa M, Nanto S, Ikeda Y, Abe K, Ogawa A. Safety and efficacy of Prasugrel in elderly/low body weight Japanese patients with ischemic stroke: randomized PRASTRO-II. Cerebrovasc Dis. 2020;49(2):152-9.

29. Wang $Y$, Chen $W$, Lin Y, Meng X, Chen G, Wang Z, Wu J, Wang D, Li J, Cao $Y$, et al. Ticagrelor plus aspirin versus clopidogrel plus aspirin for platelet reactivity in patients with minor stroke or transient ischaemic attack: open label, blinded endpoint, randomised controlled phase II trial. BMJ. 2019;365:12211.

30. Angiolillo DJ, Bernardo E, Sabate M, Jimenez-Quevedo P, Costa MA, Palazuelos J, Hernandez-Antolin R, Moreno R, Escaned J, Alfonso F, et al. Impact of platelet reactivity on cardiovascular outcomes in patients with type 2 diabetes mellitus and coronary artery disease. J Am Coll Cardiol. 2007;50(16):1541-7.

31. Gurbel PA, Bliden KP, Butler K, Tantry US, Gesheff T, Wei C, Teng R, Antonino MJ, Patil SB, Karunakaran A, et al. Randomized double-blind assessment of the ONSET and OFFSET of the antiplatelet effects of ticagrelor versus clopidogrel in patients with stable coronary artery disease: the ONSET/ OFFSET study. Circulation. 2009;120(25):2577-85.

32. Angiolillo DJ, Fernandez-Ortiz A, Bernardo E, Alfonso F, Macaya C, Bass TA, Costa MA. Variability in individual responsiveness to clopidogrel: clinical implications, management, and future perspectives. J Am Coll Cardiol. 2007; 49(14):1505-16.

33. Niijima S, Ohmori T, Kario K. Differential impact of diabetes mellitus on antiplatelet effects of prasugrel and clopidogrel. Thromb J. 2018;16:5.

34. Chouchene S, Dabboubi R, Raddaoui H, Abroug H, Ben Hamda K, Hadj Fredj S, Abderrazak F, Gaaloul M, Rezek M, Neffeti F, et al. Clopidogrel utilization in patients with coronary artery disease and diabetes mellitus: should we determine CYP2C19*2 genotype? Eur J Clin Pharmacol. 2018;74(12):1567-74.

35. Mizobe M, Hokimoto S, Akasaka T, Arima Y, Kaikita K, Morita K, Miyazaki H, Oniki K, Nakagawa K, Ogawa H. Impact of CYP2C19 polymorphism on clinical outcome following coronary stenting is more important in nondiabetic than diabetic patients. Thromb Res. 2014:134(1):72-7.

\section{Publisher's Note}

Springer Nature remains neutral with regard to jurisdictional claims in published maps and institutional affiliations. 